\title{
Test Set Enrichment using a Probabilistic Fault Model and the Theory of Output Deviations
}

\author{
Zhanglei Wang ${ }^{\dagger}$, Krishnendu Chakrabarty ${ }^{\dagger *}$, and Michael Goessel ${ }^{\ddagger}$ \\ ${ }^{\dagger}$ Dept. Electrical \& Computer Engineering \\ ${ }^{\ddagger}$ Institute of Computer Science \\ Duke University, Durham, NC 27708, USA \\ E-mail: \{zw8,krish\}@ee.duke.edu \\ University of Potsdam, Germany \\ E-mail: mgoessel@cs.uni-potsdam.de
}

\begin{abstract}
We present a probabilistic fault model that allows any number of gates in an integrated circuit to fail probabilistically. Tests for this fault model, determined using the theory of output deviations, can be used to supplement tests for classical fault models, thereby increasing test quality and reducing the probability of test escape. Output deviations can also be used for test selection, whereby the most effective test patterns can be selected from large test sets during time-constrained and highvolume production testing. Experimental results are presented to evaluate the effectiveness of patterns with high output deviations for the single stuck-at and bridging fault models.
\end{abstract}

\section{INTRODUCTION}

Due to shrinking process technologies and the use of new materials such as copper and high- $\mathrm{K}$ dielectrics for manufacturing, defects for integrated circuits (ICs) are becoming increasingly complex [1], [2]. It is not always possible to accurately model these defects or predict their occurrences. A single spot defect is likely to affect several logic gates and multiple defects on a die are likely. The locations of these defects are unpredictable. The failure probability of a gate due to a defect depends also on factors such as die temperature and power supply variations. As a result, it is no longer adequate to rely only a deterministic fault model, especially a fault model that targets only single faults. In addition, many real defects are difficult to model by existing fault models, such as resistive opens and shorts, crosstalk, and interconnect defects. Thus while test generation using classical fault models will continue to be useful [3]-[6], it is also important to focus on alternative methods for enriching test sets obtained using classical fault-oriented methods.

In this work, we consider a probabilistic fault model that allows any number of gates in the IC to fail probabilistically. Tests for this fault model, determined using the theory of output deviations, can be used to supplement tests for classical fault models, thereby increasing test quality and reducing the probability test escape. By targeting multiple fault sites in a probabilistic manner, such a model is useful for addressing phenomenon or mechanisms that are not fully understood. Output deviations can also be used for test selection, whereby the most effective test patterns can be selected from large test sets during time-constrained and high-volume production testing [7].

*The work of K. Chakrabarty was supported in part by a research fellowship from the Alexander von Humboldt Foundation, Germany.
Before presenting the main ideas underlying this work, we briefly review related prior work. The problem of ensuring reliable circuit operation in the presence of faulty components was studied in considerable detail in the past [8][11], and more recently for nanotechnology devices [12]. The key idea here is to use redundancy to ensure correct circuit outputs if every logic gate is assumed to fail with probability $\epsilon$. Elegant theoretical results have been derived on the amount of redundancy required for a given upper bound on $\epsilon$. However, these results are of limited practical value because the redundancy is often excessive, the results target only special classes of circuits, and a fault model that assigns the same failure probability to every gate (and for every input combination) is too restrictive. Stochastic techniques have also been proposed to compute reliably using logic gates that fail probabilistically [13].

More recently, a generalized deterministic fault model based on fault tuples has been developed for test generation and diagnosis [14]. Fault tuples allow us to model various types of defects in a unified manner. The detection and masking of soft errors due to single-event upsets has also received attention. Methods to reduce soft error rates and commercial tools for identifying susceptible circuit nodes and for radiation hardening are being developed [15]-[17]. Design techniques for the containment of soft errors have also been developed [18]. Probabilistic transfer matrices have been used for generating test patterns for soft errors caused by singleevent upsets [19]. The focus of this work however is on test set enrichment using a probabilistic fault model to detect unmodeled permanent faults. We use this fault model and the concept of output deviations to select vectors that are likely to be more effective in detecting unmodeled faults. To evaluate this method, non-feedback single/multiple bridging faults are used to mimic unmodeled faults. Experimental results show that vectors selected using the probabilistic fault model and output deviations provide higher fault coverage.

The remainder of this paper is organized as follows. Sections II-IV introduce the probabilistic fault model and the theory of output deviations. Design of experiments and some experimental results are reported in Section V. Section VI concludes this paper and outlines directions for future work.

\section{Modeling of GATE RELIABility}

In this section, we define the reliability of a logic gate and show how it can be determined using transistor-level 
schematics.

The reliability of a single-output gate encompasses all the different input combinations of the gate, and for a given input combination, it provides the probability that the gate output is correct for the corresponding input combination. The probability that a gate output is correct can be different for the various input combinations. Formally, we defi ne the reliability of a gate with $m$ inputs and one output by a $2^{m}$-dimensional vector.

Definition 1: The reliability $R_{i}$ of a gate $G_{i}$ with $m$ inputs and a single output is a vector with $2^{m}$ components, defi ned as:

$$
R_{i}=\left(r_{i}^{(00 \ldots 00)} r_{i}^{(00 \ldots 01)} r_{i}^{(00 \ldots 10)} \ldots r_{i}^{(11 \ldots 11)}\right),
$$

where each component of $R_{i}$ denotes the probability that the gate output is correct for the corresponding input combination.

For example, $r_{i}^{00}$ is the probability that the output of a 2input $G_{i}$ is correct under input 00 . If $m=2$ for a logic gate, we have $R_{i}=\left(r_{i}^{(00)} r_{i}^{(01)} r_{i}^{(10)} r_{i}^{(11)}\right)$.

The above gate-level reliability vectors can be generated in a number of ways, e.g., using layout information, inductive fault analysis [20], and failure data analysis. It can also be estimated using simple transistor-level failure probabilities. Our objective here is not to develop new techniques for determining reliability vectors, but rather to use these as inputs for the computation of output deviation. In practice, deviationbased test patterns can be generated using multiple sets of reliability vector estimates and the probabilistic fault model.

Consider the 2-input NAND and NOR gates shown in Fig. 1. Suppose each transistor can be stuck-open due to a defect, i.e., it cannot be switched on, with probability $\alpha$. Similarly, suppose each transistor can be stuck-on due to a defect, i.e., it cannot be switched off, with probability $\beta$. Next let us consider input combination $x_{1} x_{2}=00$. If only stuck-on faults are considered, the NAND gate produces the correct output for this combination with probability $1-\alpha^{2}$ because the gate produces an incorrect output only if both transistors are stuckopen. (The absence of a second pull-up path affects the pullup time for this input combination but this issue is ignored since worst-case pull-up times consider only one pull-up path.) Likewise, if we only consider stuck-on faults, the probability that the NAND gate produces the correct output for input 00 is $1-2 \beta+\beta^{2}$.

Table I presents the reliabilities of the NAND and NOR gates for stuck-open and stuck-on failure modes $\left(R_{1}^{N A N D}\left(\tilde{x_{1}}, \tilde{x_{2}}, \alpha\right)\right.$ and $R_{2}^{N A N D}\left(\tilde{x_{1}}, \tilde{x_{2}}, \beta\right)$, respectively) for different input combinations. Note that $\tilde{x_{1}}$ and $\tilde{x_{2}}$ refer to input combinations, e.g., $R_{1}^{N A N D}(0,0, \alpha)$ refers to the probability that input 00 produces the correct output when the stuck-on probability for a transistor is $\alpha$. If the stuck-open or stuck-on probabilities for the different transistors are unequal, e.g., due to different device dimensions, the actual values can be easily used to calculate the reliabilities in Table I. (We assume that the gate output is in error if both pull-up and pull-down paths are active.)

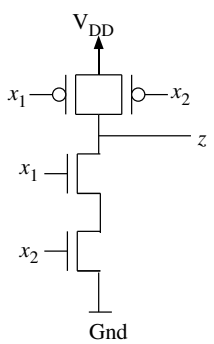

(a)

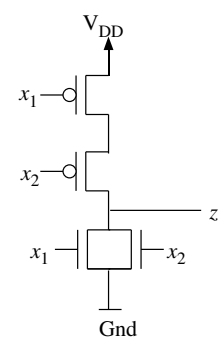

(b)
Fig. 1. 2-input NAND and NOR gates in static ratioless CMOS technology.

$\mid$\begin{tabular}{||c|c|c||}
\hline$x_{1} x_{2}$ & $R_{1}^{N A N D}\left(\tilde{x_{1}}, \tilde{x_{2}}, \alpha\right)$ & $R_{2}^{N A N D}\left(\tilde{x_{1}}, \tilde{x_{2}}, \beta\right)$ \\
\hline 00 & $1-\alpha^{2}$ & $1-\beta^{2}$ \\
01 & $1-\alpha$ & $1-\beta$ \\
10 & $1-\alpha$ & $1-\beta$ \\
11 & $1-2 \alpha+\alpha^{2}$ & $1-2 \beta+\beta^{2}$ \\
\hline$x_{1} x_{2}$ & $R_{1}^{N O R}\left(\tilde{x_{1}}, \tilde{x_{2}}, \alpha\right)$ & $R_{2}^{N O R}\left(\tilde{x_{1}}, \tilde{x_{2}}, \beta\right)$ \\
\hline 00 & $1-2 \alpha+\alpha^{2}$ & $1-2 \beta+\beta^{2}$ \\
01 & $1-\alpha$ & $1-\beta$ \\
10 & $1-\alpha$ & $1-\beta$ \\
11 & $1-\alpha^{2}$ & $1-\beta^{2}$ \\
\hline \multicolumn{2}{|c|}{ (b) }
\end{tabular}

TABLE I

RELIABILITIES OF THE NAND AND NOR GATES EXPRESSED IN TERMS OF TRANSISTOR STUCK-ON AND STUCK-OPEN PROBABILITIES.

Let $R\left(\tilde{x_{1}}, \tilde{x_{2}}, \alpha, \beta\right)$ be the gate reliability for input combination $\left(\tilde{x}_{1}, \tilde{x_{2}}\right)$, when any number of stuck-open and stuckon faults can simultaneously occur. It can be easily shown that $R\left(\tilde{x_{1}}, \tilde{x_{2}}, \alpha, \beta\right)=R_{1}\left(\tilde{x_{1}}, \tilde{x_{2}}, \alpha\right) \cdot R_{2}\left(\tilde{x_{1}}, \tilde{x_{2}}, \beta\right)$. The result follows from the fact that for any input combination, the set of transistors that affects the output under stuck-on conditions is disjoint from the set of transistors that affects the output under stuck-open conditions.

In this paper, we limit ourselves to basic gates with one or two inputs. Extension of the theory of output deviations to gates with larger fanout is straightforward. Here, gates with larger fanout are fi rst expanded to a network of basic gates.

\section{COMPUTATION OF SIGNAL PROBABILITIES}

Next we associate signal probabilities $p_{i, 0}$ and $p_{i, 1}$ with each line $i$ in the circuit, where $p_{i, 0}$ and $p_{i, 1}$ are the probabilities for line $i$ to be at logic 0 and 1 , respectively. Obviously we have $p_{i, 0}+p_{i, 1}=1$. The calculation of the signal probabilities is along the same lines as introduced in [21], and used later in [22]. To reduce the amount of computation as in [21], [22], signal correlations due to reconvergent fanout are not considered here.

Let $i$ be the output of a two-input gate $G$. Let $j$ and $k$ denote the input lines for this gate. If $G$ is a NAND gate, we have:

$$
\begin{aligned}
p_{i, 0} & =p_{j, 1} p_{k, 1} r_{i}^{(11)}+p_{j, 0} p_{k, 0}\left(1-r_{i}^{(00)}\right) \\
& +p_{j, 0} p_{k, 1}\left(1-r_{i}^{(01)}\right)+p_{j, 1} p_{k, 0}\left(1-r_{i}^{(10)}\right) \\
p_{i, 1} & =p_{j, 0} p_{k, 0} r_{i}^{(00)}+p_{j, 0} p_{k, 1} r_{i}^{(01)}
\end{aligned}
$$


(0.9 0.90 .90 .8$)$

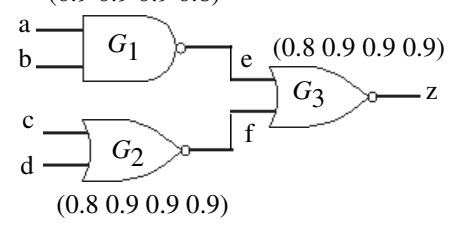

Fig. 2. An illustrative example.

\begin{tabular}{||c|c|c|c|c|c|c||}
\hline Input pattern, $z$ & $p_{e, 0}$ & $p_{e, 1}$ & $p_{f, 0}$ & $p_{f, 1}$ & $p_{z, 0}$ & $p_{z, 1}$ \\
\hline 0000,0 & 0.1 & 0.9 & 0.2 & 0.8 & 0.886 & 0.114 \\
0101,0 & 0.1 & 0.9 & 0.9 & 0.1 & 0.837 & 0.163 \\
1111,1 & 0.8 & 0.2 & 0.9 & 0.1 & 0.396 & 0.604 \\
\hline
\end{tabular}

TABLE II

SIGNAL PROBABILITIES FOR DIFFERENT INPUT COMBINATIONS

$$
+p_{j, 1} p_{k, 0} r_{i}^{(10)}+p_{j, 1} p_{k, 1}\left(1-r_{i}^{(11)}\right)
$$

The above defi nition of the signal probabilities can be easily extended to the case of more than two inputs. It can also be easily verifi ed that $p_{i, 0}+p_{i, 1}=p_{j, 0} p_{k, 0}+p_{j, 0} p_{k, 1}+p_{j, 1} p_{k, 0}+$ $p_{j, 1} p_{k, 1}=1$.

Let $G$ be a gate with two inputs $j$ and $k$, controlling value $c$, and inversion value $v$. For example, a NAND gate has controlling value $c=0$ and inversion value $v=1$ [23]. Let $\bar{c}$ be the complement of the controlling value $c$. The signal probabilities for the output $i$ of such a gate can be easily expressed as follows:

$$
\begin{aligned}
p_{i, c \oplus v} & =p_{j, c} p_{k, c} r_{i}^{(c c)}+p_{j, c} p_{k, \bar{c}} r_{i}^{(c \bar{c})} \\
& +p_{j, \bar{c}} p_{k, c} r_{i}^{(\bar{c} c)}+p_{j, \bar{c}} p_{k, \bar{c}}\left(1-r_{i}^{(\bar{c} c)}\right) \\
p_{i, \bar{c} \oplus v} & =p_{j, c} p_{k, c}\left(1-r_{i}^{(c c)}\right)+p_{j, c} p_{k, \bar{c}}\left(1-r_{i}^{(c \bar{c})}\right) \\
& +p_{j, \bar{c}} p_{k, c}\left(1-r_{i}^{(\bar{c} c)}\right)+p_{j, \bar{c}} p_{k, \bar{c}} r_{i}^{(\bar{c} c)}
\end{aligned}
$$

Next consider a gate without a controlling value, e.g., XOR and XNOR. For the XOR gate, the signal probabilities can be expressed as follows (the formulas for the XNOR gate are similar):

$$
\begin{aligned}
p_{i, 0} & =p_{j, 0} p_{k, 0} r_{i}^{(00)}+p_{j, 0} p_{k, 1}\left(1-r_{i}^{(01)}\right) \\
& +p_{j, 1} p_{k, 0}\left(1-r_{i}^{(10)}\right)+p_{j, 1} p_{k, 1} r_{i}^{(11)} \\
p_{i, 1} & =p_{j, 0} p_{k, 0}\left(1-r_{i}^{(00)}\right)+p_{j, 0} p_{k, 1} r_{i}^{(01)} \\
& +p_{j, 1} p_{k, 0} r_{i}^{(10)}+p_{j, 1} p_{k, 1}\left(1-r_{i}^{(11)}\right)
\end{aligned}
$$

Figure 2 shows a simple circuits consisting of three gates $G_{1}, G_{2}$, and $G_{3}$ with reliability vectors (0.9 0.90 .90 .8$)$, (0.8 0.90 .90 .9 ), and (0.8 0.90 .90 .9 ), respectively. For the three different deterministic input vectors 0000,010 , and 1111, the signal probabilities are determined and presented in Table II. The fault-free values at the output $z$ are also listed in the first column of Table II.

In the next section, we use the concept of signal probabilities to develop the notion of output deviations. We then describe how to determine deviation-based test patterns that can be used to enrich test sets.

\begin{tabular}{||c|c|c|c||}
\hline $\begin{array}{c}\text { Fault } \\
\text { event }\end{array}$ & Fault event description & Event probability & $\begin{array}{c}\text { Output } \\
\text { value }\end{array}$ \\
\hline $\mathcal{E}_{0}$ & $G_{1}, G_{2}, G_{3}$ fault-free & $.9 \times .8 \times .9=.648$ & 0 \\
\hline $\mathcal{E}_{1}$ & $G_{1}, G_{2}$ fault-free, $G_{3}$ faulty & $.9 \times .8 \times .1=.072$ & 1 \\
\hline $\mathcal{E}_{2}$ & $G_{1}, G_{3}$ fault-free, $G_{2}$ faulty & $.9 \times .2 \times .9=.162$ & 0 \\
\hline $\mathcal{E}_{3}$ & $G_{2}, G_{3}$ fault-free, $G_{1}$ faulty & $.1 \times .8 \times .9=.072$ & 0 \\
\hline $\mathcal{E}_{4}$ & $G_{1}, G_{2}$ faulty, $G_{3}$ fault-free & $.1 \times .2 \times .8=.016$ & 1 \\
\hline $\mathcal{E}_{5}$ & $G_{1}, G_{3}$ faulty, $G_{2}$ fault-free & $.1 \times .8 \times .1=.008$ & 1 \\
\hline $\mathcal{E}_{6}$ & $G_{2}, G_{3}$ faulty, $G_{1}$ fault-free & $.9 \times .2 \times .1=.018$ & 1 \\
\hline $\mathcal{E}_{7}$ & $G_{2}, G_{3}, G_{1}$ faulty & $.1 \times .2 \times .2=.004$ & 0 \\
\hline
\end{tabular}

TABLE III

FAULT EVENTS FOR THE CIRCUIT OF FIG. 2 UNDER INPUT PATTERN $a b c d=0000$.

\section{OutPut DEViations AND testing}

For any logic gate (or primary output) $g$ in a circuit, let its fault-free output value for any given input pattern $t_{j}$ be $d$, $d \in\{0,1\}$. The output deviation $\Delta_{g, j}$ of $g$ for input pattern $t_{j}$ is defi ned as $p_{g, \bar{d}}$, where $\bar{d}$ is the complement of $d$. Intuitively, the deviation for an input pattern is a measure of the likelihood that the gate output is incorrect for that input pattern.

Next we formally defi ne the probabilistic fault model for a combinational circuit $\mathcal{C}$, and its application in testing.

Definition 2: A combinational circuit $\mathcal{C}$ is defi ned as $\mathcal{C}=$ $\{\mathcal{G}, \mathcal{P} \mathcal{I}, \mathcal{Z}, \mathcal{R}\}$, where $\mathcal{G}=\left\{G_{1}, G_{2}, \ldots, G_{N}\right\}$ is the set of logic gates in $\mathcal{C}, \mathcal{P} \mathcal{I}$ and $\mathcal{Z}$ are the sets of primary inputs and outputs respectively, and $\mathcal{R}=\left\{R_{1}, R_{2}, \ldots, R_{N}\right\}$ is the set of reliabilities of the gates in $\mathcal{G}$.

Definition 3: A probabilistic fault model $\mathcal{F}$ for circuit $\mathcal{C}$ is defi ned as follows. Each gate $G_{i}$ can fail independently of other gates and its fault behavior is defi ned by $R_{i}$.

Under this fault model, the expected output values of the circuit in response to an input pattern is no longer deterministic. Rather, it is given by the signal probabilities at primary outputs. Note that the circuit behavior is assumed to be deterministic after manufacturing; the probabilistic fault model is only used during test development to generate tests for defects that occur in a random fashion, and which cannot be easily modeled.

Consider the simple circuit shown in Fig. 2. According to $\mathcal{F}$, this circuit can fail in a number of ways, each of which is termed a fault event. Table III lists the various fault events $\mathcal{E}_{1}, \mathcal{E}_{2}, \ldots \mathcal{E}_{7}$ and the event $\mathcal{E}_{0}$, corresponding to the fault-free case. It also lists the probability associated with each fault event, and the corresponding circuit output, for input pattern $a b c d=0000$. Only the events $\mathcal{E}_{1}, \mathcal{E}_{4}, \mathcal{E}_{5}$, and $\mathcal{E}_{6}$ are detected by the given input pattern. Let $\mathcal{E}$ be the event that the pattern 0000 detects a fault in the circuit. It can be easily seen that

$$
\begin{aligned}
P[\mathcal{E}] & =P\left[\mathcal{E}_{1} \bigcup \mathcal{E}_{4} \bigcup \mathcal{E}_{5} \bigcup \mathcal{E}_{6}\right] \\
& =P\left[\mathcal{E}_{1}\right]+P\left[\mathcal{E}_{4}\right]+P\left[\mathcal{E}_{5}\right]+P\left[\mathcal{E}_{6}\right] \\
& =0.114
\end{aligned}
$$

since the fault events are mutually exclusive. Note from Table II that $\Delta_{z}$ for input pattern 0000 is also 0.114 .

The above example shows that the probability that an input pattern detects a fault can be calculated by enumerating the fault events. However, signal probability and deviation 
calculations are sufficient to determine the fault detection probability. This observation is formalized by the following theorem, the proof of which is omitted due to lack of space.

Theorem 1: Let $\mathcal{E}$ be the event that input pattern $t_{j}$ detects a fault in a circuit $\mathcal{C}$ at output $z_{i}$ under fault model $\mathcal{F}$. Then $P[\mathcal{E}]=\Delta_{z_{i}, j}$.

The probability that $t_{j}$ will produce an observable error at $z_{i}$ for fault model $\mathcal{F}$ is directly proportional to $\Delta_{z_{i}, j}$. Therefore, a goal of testing is to apply those vectors to $\mathcal{C}$ that produce large deviations at the output. These tests can be used to supplement tests for standard fault models. From now on we only consider output deviations at outputs and use the terms "output deviation" and "deviation" interchangeably. In the next section, we experimentally study the fault detection capabilities of sets of patterns with different deviation values.

\section{EXPERIMENTAL RESUlTS}

We use two arbitrarily chosen sets of reliability vectors for our experiments. These vectors are defined separately for each gate type. For example, for a 2-input NAND gate, we use 1) "low reliability" vector: $R^{N A N D 2}=$ $\left(0.8^{(00)}, 0.8^{(01)}, 0.8^{(10)}, 0.7^{(11)}\right)$, and 2$)$ "high reliability" vector: $R^{N A N D 2}=\left(0.95^{(00)}, 0.95^{(01)}, 0.95^{(10)}, 0.85^{(11)}\right)$. The steps in these experiments are as follows:

Step 1: Generate a set of target faults $F_{\text {target }}$.

Step 2: Generate a set of random vectors $T_{\text {rand }}$.

Step 3: Compute deviations for the vectors in $T_{\text {rand }}$.

Step 4: Perform fault simulation on $F_{\text {target }}$ using $T_{\text {rand }}$. Add detected faults to the set $F_{\text {det }}$.

Step 5: Use a predetermined threshold $\gamma$ on the output deviation value to divide $T_{\text {rand }}$ into two sets $T_{\text {high }}$ and $T_{\text {low }}$. Vectors that lead to a deviation greater than the threshold $\gamma$ on at least one primary output are placed in $T_{h i g h}$. The other vectors are placed in $T_{\text {low }}$.

Step 6: Determine the fault coverages for $F_{\text {det }}$ using $T_{\text {high }}$ and $T_{l o w}$. These fault coverages are denoted by $F C_{h i g h}$ and $F C_{\text {low }}$, respectively. To allow fair comparison involving fault coverages, the larger test set is randomly truncated such that the two test sets $T_{\text {high }}$ and $T_{\text {low }}$ have the same number of vectors.

In the following set of experiments (numbered as 1, 2, 3, 4), we use single stuck-line (SSL) faults, single non-feedback bridging-faults (s-NFBFs), and double non-feedback bridgingfaults (double-NFBFs), as the types of faults in $F_{\text {target }}$. Each double-NFBF contains 2 s-NFBFs. Moreover, we ensure that $F_{\text {target }}$ only contains faults that are not detected by an SSL test set generated by Atalanta [24] with random-fi 11 turned on For simplicity, in this section $F_{\text {target }}(i)$ refers to $F_{\text {target }}$ used in Experiment $i$. $T_{\text {rand }}$ contains 9,704 vectors in Experiments 1, 2 and 4, and 48,454 vectors in Experiment 3.

Experimental results show that $F C_{h i g h}$ is significantly higher than $F C_{\text {low }}$ for most ISCAS' 85 and ISCAS' 89 benchmark circuits. Due to limited space, we only include representative results for selected benchmarks.
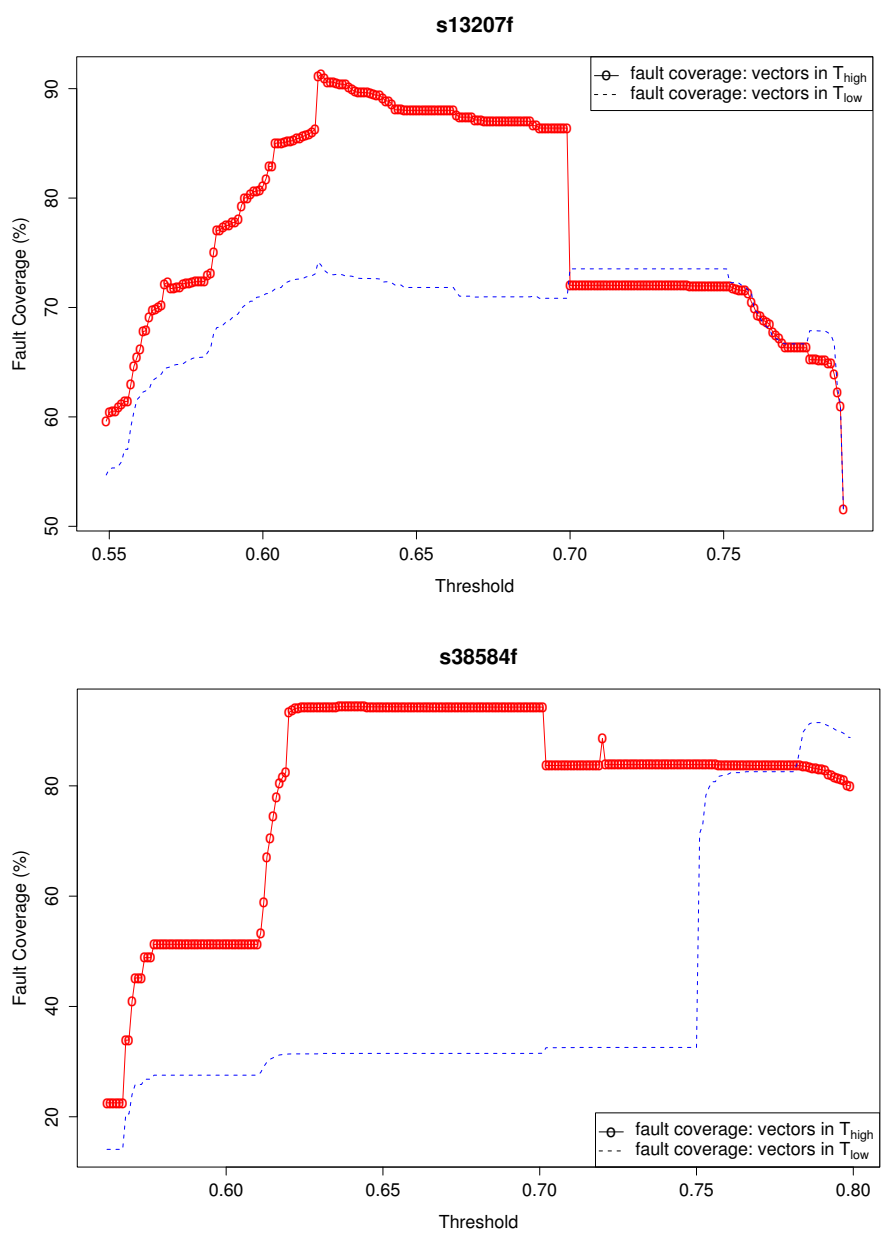

Fig. 3. Fault coverage obtained using $T_{\text {high }}$ and $T_{\text {low }}$ for the SSL faults, with "high reliability" vector for the gates.

\section{Experiment 1: SSL fault coverage}

$F_{\text {target }}(1)$ contains all SSL faults in the CUT. Fig. 3 shows results for s 13207 and s38584 with the "high reliability" vector for the gates. The $\mathrm{x}$-axis corresponds to a range of values for the threshold $\gamma$. The $\mathrm{y}$-axis indicates the fault coverage obtained using $T_{\text {high }}$ and $T_{\text {low }}$.

\section{Experiment 2: $s$-NFBF fault coverage}

To generate $F_{\text {target }}(2)$, we fi rst enumerate all s-NFBFs in the CUT for smaller benchmarks and a sampled set of sNFBFs for the larger benchmarks. We consider wired-AND and wired-OR shorts in this experiment. Next faults detected by the SSL test set are removed from further consideration. In this way, we are able to demonstrate that high-deviation patterns can enrich test sets for fault detection. Fig. 4 shows the results for s13207 and s38584.

\section{Experiment 3: double-NFBF fault coverage}

To generate $F_{\text {target }}(3), F_{\text {target }}(2)$ is used as a repository, from which 10,000 double-NFBFs are randomly generated. Again, to demonstrate test set enrichment, double-NFBFs detected by the SSL test set are removed. Fig. 5 shows the fault coverage results for c7552 and s38584. 

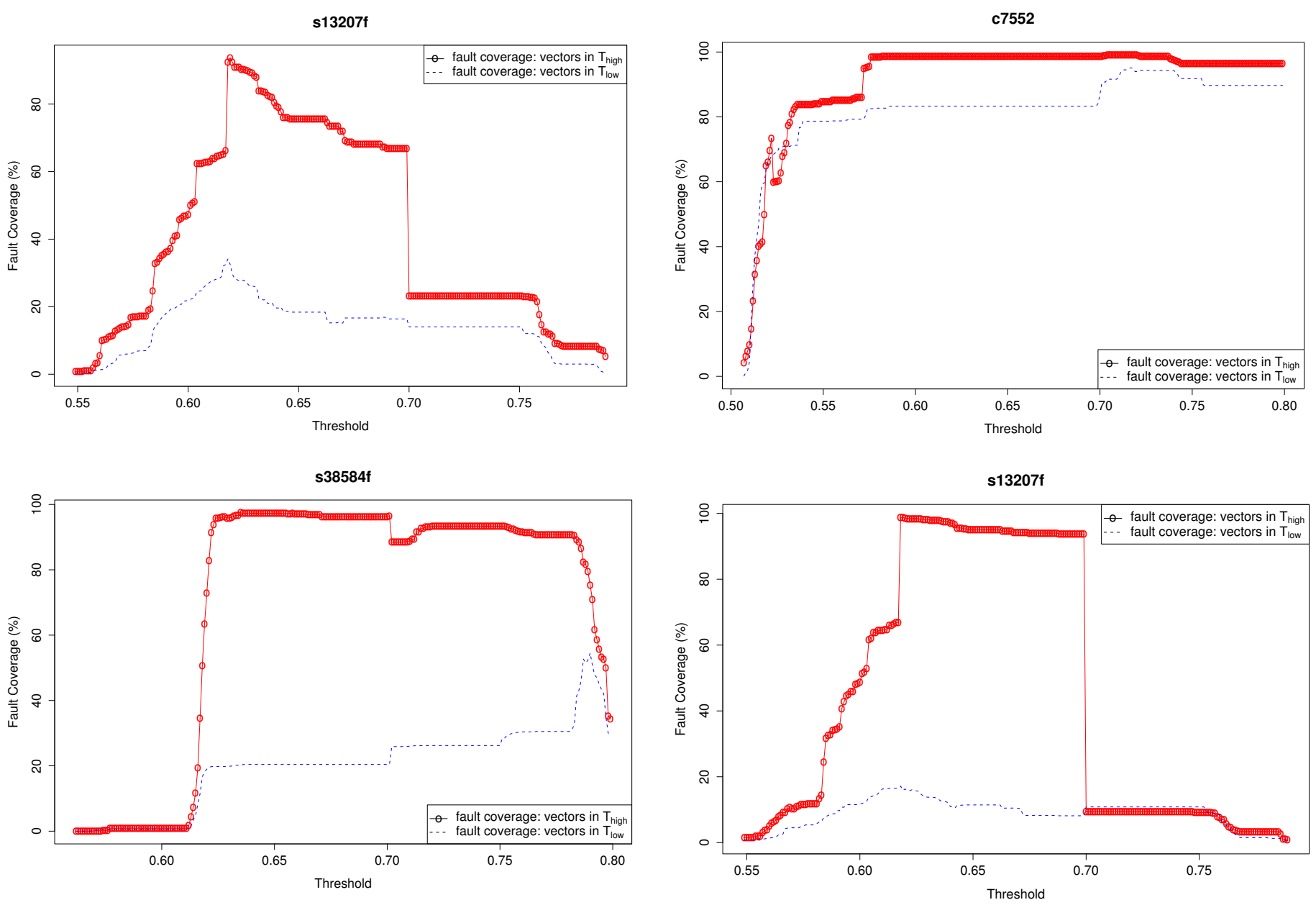

Fig. 4. Fault coverage obtained using $T_{h i g h}$ and $T_{l o w}$ for the s-NFBF faults, with "high reliability" vector for the gates.

\section{Experiment 4: Restricted Neighborhood Mode}

A special case of the probabilistic fault model can be viewed as a restricted neighborhood mode. Here we assume a single defect site and impose the condition that gates in the neighborhood of this site are probabilistically faulty. A neighborhood is a set of gates in the vicinity of the gate that corresponds to the defect site. The size of a neighborhood is referred to as the radius $r$ of the restricted neighborhood mode. Logic gates outside this neighborhood have reliability vectors will all their components equal to 1 . This mode is useful in practice when prior information about potential defect locations in a chip is available. It is also useful during "targeted" test generation and test selection.

Information about neighborhoods can be extracted from layout data. In this paper, we only consider $r=0$ and $r=1$; in particular, we assume that the neighborhood consists of the defect site $G$ and gates that are at most one-level away (upstream as well as downstream) from $G$. (Experiments 1-3 can therefore be viewed as a special case with $r=\infty$.) We calculate deviations for $r=0,1, \infty$. Fig. 6 shows the results obtained for s38584.

We can see from Experiments 1-4, and from the results for other benchmark circuits that could not be included due to lack

Fig. 5. Fault coverage obtained using $T_{\text {high }}$ and $T_{\text {low }}$ for the double-NFBF faults, with "high reliability" vector for the gates.

of space, that for most circuits, the fault coverage is higher for a set of vectors with high output deviation. Moreover, the choice of the threshold $\gamma$ appears to be important. The best threshold value seems to be circuit-dependent.

The program to compute output deviations was implemented using $\mathrm{C}++$. On a Pentium $4 \mathrm{PC}$ with $2.6 \mathrm{Ghz}$ processor and $1 \mathrm{G}$ memory running Linux, it takes approximately 80 seconds to compute the deviations for 10,000 vectors for full scan version of the largest benchmark circuit (s38584). Event-driven techniques can be used to decrease computation time; for consecutive vectors, only the gates whose outputs are changed need to be evaluated.

\section{CONCLUSIONS}

We have described a probabilistic fault model that allows any number of gates in an integrated circuit to fail probabilistically. We have shown that tests for this fault model, determined using the theory of output deviations, can be used to supplement tests for classical fault models. This can potentially increase test quality and reduce test escapes. We have shown that output deviations can be used as a simple criterion to rank test patterns in terms of their effectiveness. Experimental results for benchmark circuits show that test sets with higher deviation lead to higher fault coverage for single 
stuck-at and bridging faults.

The results presented in this paper open a number of interesting research directions. Experiments involving realistic defects such as resistive shorts and opens, as well as delay faults will shed more light on the effectiveness of the probabilistic fault model and the use of output deviations as a pattern grading metric. A deviation-based test generation tool will be useful to generate vectors with high output deviations. The propagation of deviations in the presence of unknown logic values (Xs) also needs to be investigated. Finally, the theory of deviations for sequential circuits is of interest, especially for designs that do not rely on full scan.

\section{REFERENCES}

[1] J. Segura and C. F. Hawkins, CMOS Electronics : How It Works, How It Fails. Wiley-IEEE Press, 2004.

[2] R. Aitken, "New defect behavior at 130nm and beyond," in Proc. European Test Symposium, 2004, pp. 279-284.

[3] F. Corno, P. Prinetto, M. Rebaudengo, and M. S. Reorda, 'GATTO: a genetic algorithm for automatic test pattern generation for large synchronous sequential circuits," IEEE Trans. CAD, p. 943, Aug. 1996.

[4] P. Girard, C. Landrault, S. Pravossoudovitch, and B. Rodriguez, "A diagnostic ATPG for delay faults based on genetic algorithms," in Proc. IEEE Intl. Test Conf. on Test and Design Validity, 1996, pp. 286-293.

[5] Q. Xu and N. Nicolici, 'Delay fault testing of core-based systems-ona-chip," in Proc. DATE Conf., 2003, pp. 744-749.

[6] I. Pomeranz and S. M. Reddy, 'Pattern sensitivity: A property to guide test generation for combinational circuits," in Proc. 8th Asian Test Symp., 1999, pp. 75-80.

[7] Y. Tian, M. Mercer, W. Shi, and M. Grimaila, "An optimal test pattern selection method to improve the defect coverage," in Proc. Int. Test Conf., 2005.

[8] J. von Neumann, 'Probabilistic logics and the synthesis of reliable organisms from unreliable components," in C. E. Shannon and J. McCarthy, ed., Automata Studies. Princeton University Press, Princeton, NJ, 1956, pp. 43-98.

[9] R. L. Dobrushin and S. I. Ortyukov, 'Lower bound for the redundancy of self-correcting arrangements of unreliable components," Problems in Information Transmission (Translated from Problemy Peredachi Informatsii, in Russian), vol. 13, pp. 59-65, 1977.

[10] — - 'Upper bound on the redundancy of self-correcting arrangements of unreliable components," Problems in Information Transmission (Translated from Problemy Peredachi Informatsii, in Russian), vol. 13, pp. 203-218, 1977.

[11] N. Pippenger, G. D. Stamoulis, and J. N. Tsitsiklis, "On a lower bound for the redundancy of reliable networks with noisy gates," IEEE Trans. on Information Theory, vol. 37, pp. 639-643, 1991.

[12] J. Han and P. Jonker, "A system architecture solution for unreliable nanoelectronic devices," IEEE Trans. on Nanotechnology, vol. 1, pp. 201-208, December 2002.

[13] C. L. Janer et al., 'Fully parallel stochastic computation architecture," IEEE Trans. on Signal Processing, vol. 44, pp. 2110-2117, August 1996.

[14] K. N. Dwarakanath and R. D. Blanton, "Universal fault simulation using fault tuples," in Proc. Design Automation Conf., 2000, pp. 786-789.

[15] P. Shivakumar et al., "Modeling the effect of technology trends on the soft error rate of combinational logic," in Proc. Int. Conf. Dependable Systems and Networks, 2002, pp. 389-398.

[16] K. Mohanram and N. A. Touba, "Cost-effective approach for reducing soft error failure rate in logic circuits," in Proc. Int. Test Conf., 2003.

[17] White Paper: New trends and solutions to combat the soft error threat to ICs in 2004, iRoC Technologies, www.iroc.com, 2004.

[18] S. Mitra, N. Seifert, M. Zhang, Q. Shi, and K. S. Kim, 'Robust system design with built-in soft-error resilience," IEEE Computer, pp. 43-52, Feb. 2005.

[19] S. Krishnaswamy, I. L. Markov, and J. P. Hayes, 'Logic circuit testing for transient faults," in Digest of Papers of the European Test Symposium, 2005, pp. 102-107.

[20] F. J. Ferguson and J. P. Shen, "A CMOS fault extractor for inductive fault analysis," IEEE Trans. CAD, vol. 7, pp. 1181-1194, Nov. 1988.
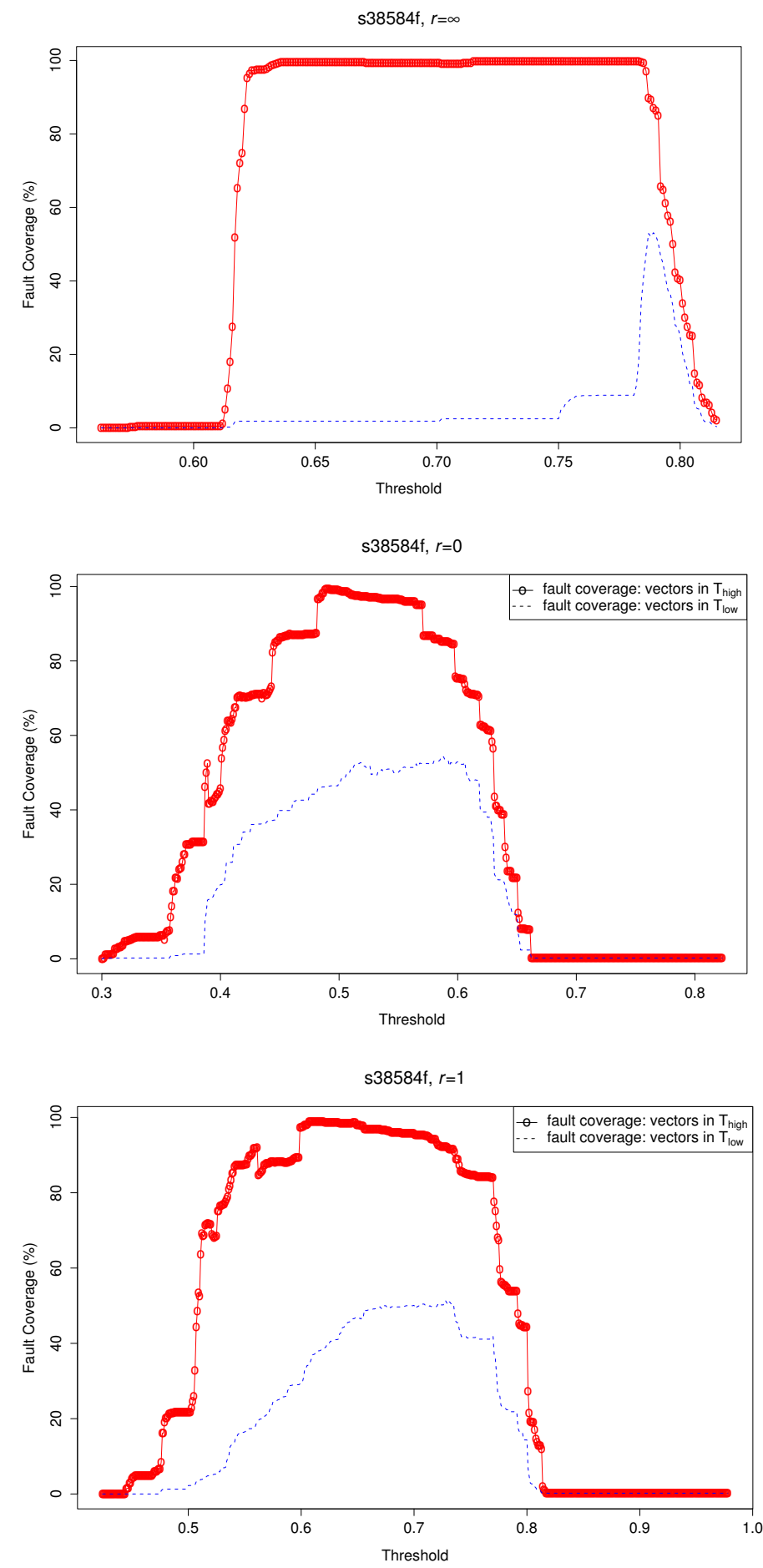

Fig. 6. Fault coverage obtained using $T_{h i g h}$ and $T_{l o w}$ for the s-NFBF faults, with the restricted neighborhood mode and "high reliability" vector.

[21] K. P. Parker and E. J. McCluskey, 'Probablistic treatment of general combinational networks," IEEE Trans. Computers, pp. 668-670, June 1975.

[22] S. C. Seth, L. Pan, and V. D. Agrawal, 'PREDICT-Probabilistic estimation of digital circuit testability," in Proc. Int. Symp. Fault-Tolerant Computing, 1985, pp. 220-225.

[23] M. Abramovici, M. A. Breuer, and A. D. Friedman, Digital Systems Testing and Testable Design. IEEE Press, Piscataway, NJ, 1990.

[24] H. K. Lee and D. S. Ha, 'On the generation of test patterns for combinational circuits,” Dep’t of Electrical Eng., Virginia Polytechnic Institute and State University, Tech. Rep. 12_93, 1993. 